\title{
Targeted therapy of glucose uptake via GLUT1 kills RCC cells
}

Renal cell carcinoma (RCC) is resistant to both radiation therapy and chemotherapy, and over $90 \%$ of patients harbor tumors that are also unresponsive to immunotherapy. Thus, curative therapies are urgently needed for patients with advanced forms of RCC.

A study by Amato Giaccia and colleagues has shown that a synthetic drug named STF-31 selectively targets the glucose transporter GLUT1 in RCC cells to inhibit glucose uptake, thereby starving the cells of their energy supply and resulting in necrotic cell death. As they explain in their paper in Science Translational Medicine,

"The GLUT1-selective cytotoxicity induced by [this drug] provides direct evidence that many cancer cell types are dependent on glycolysis."

The team used RCC4, an RCC cell line that lacks the von Hippel-Lindau (VHL) tumor suppressor gene, to screen more than 60,000 small molecules, identifying STF-31 as a lethal compound that selectively kills VHL-deficient cells. Interestingly, hypoxia-inducible factor, a transcription factor involved in adaptation to anaerobic conditions, was found to be implicated in STF-31-induced cytotoxicity in these cells. Furthermore, STF-31 treatment of RCC4 cells reduced lactate production, suggesting that STF-31induced cytotoxicity involves inhibition of glycolysis. Indeed, using a radioactive glucose analogue, the researchers demonstrated that STF-31 treatment significantly inhibited glucose uptake in RCC4 cells, and ATP production was decreased by $75 \%$.

Further experiments revealed that STF-31 inhibits glucose uptake through direct interaction with GLUT1, the most abundant glucose transporter in RCC cells. Knock down of GLUT1 resulted in substantial cell death, which together indicate that STF-31-induced cytotoxicity in RCC occurs via selective GLUT1 inhibition. Data from animal experiments mirrored the results of the in vitro studies, with positron emission tomography showing that subcutaneous inoculation of soluble STF-31 into mice with VHLdeficient human RCCs markedly reduced glucose uptake by the tumor cells. Notably,

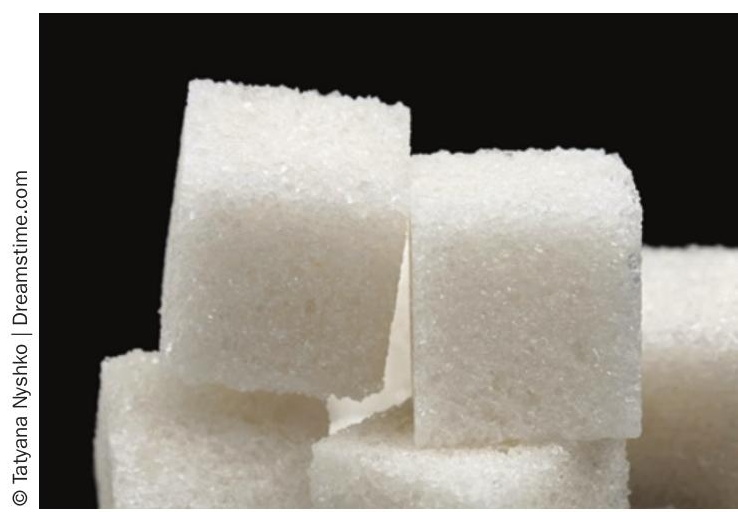

no toxic effects were observed in normal tissue, including immune cells or the brain.

"[STF-31] inhibits the growth of RCCs by binding GLUT1 directly and impeding glucose uptake in vivo without toxicity to normal tissue," the authors comment in their report. "Our data show that the effectiveness of [this drug] can be monitored by in vivo imaging." Giaccia and colleagues now intend to test STF-31 in clinical trials, in order to determine whether GLUT1 inhibition could provide a therapeutic target for advanced RCC.

Iley Ozerlat

Original article Chan, D. A. et al. Targeting GLUT1 and the Warburg effect in renal cell carcinoma by chemical synthetic lethality. Sci. Transl. Med. 3, 94ra70 (2011) 